\title{
Measurement of Natural Properties of a Beam Based on Machine Vision
}

\author{
Qingpeng Han ${ }^{1 a}$, Peixin $\mathrm{Gao}^{2 \mathrm{~b}}$, Ling $\mathrm{Tang}^{2}$, Xiaowei Sun ${ }^{2}$, Zhong Luo ${ }^{2 \mathrm{c}}$ \\ ${ }^{1}$ Liaoning University of Science and Technology, Anshan, Liaoning114051, China \\ ${ }^{2}$ Northeastern University, Shenyang 110819, China \\ ahan1011@163.com, bpeter19870923@163.com, czhluo@mail.neu.edu.cn
}

Keywords: flexible beam, machine vision, natural properties

Abstract. The natural properties of flexible beam is measured by using machine vision in which the image of vibrate flexible Beam is acquired continuously. Different from the previous contact measurement methods, the binary processing method and centroid method is utilized to binarize the image and extracts the position of marked point. Gathering and calculating the position information preprocessed in MATLAB indicates the natural properties of flexible beam. Furthermore, the test results compared with the theoretical analysis verifies the validity of the proposed method.

\section{Introductions}

The conventional electrical test sensors are of great interests in many fields of industry, especially for vibration measurement. However, with low precision, big error and perplexing to operate, several non-contact methods that overcome the above defects have been introduced in the past several years. One of the testing methods based on CCD image sensor has become one of the important directions at the present $[1,2]$. The image measurement based on optics, syncretized electronics, computer technology, laser science and image processing forms a complex optical, electro mechanical measurement system.

Subjected to speed impact or in the process of motion, the flexible beam would produce vibration. CCD, with high accuracy, high efficiency and automatic degree to test the natural properties of flexible beam, has becoming more of a concern. The optical imaging principle of CCD is to put the object image on imaging device which has photoelectric conversion function by optical system [3], and then the location of the object is recorded. The binary processing method and centroid method is used to binarize the image and extracts the position of marked point. The time domain response of the flexible beam, waterfall diagram, frequency, damping ratio by calculating and processing the data are used to verify the feasibility of the proposed test method.

\section{Test system}

The principle diagram of flexible beam natural properties test based on CCD is shown Fig. 1. The test system consist of optical imaging part, data acquisition part, data transmission part, date processing part, and the output and storage part.

The light source sends out uniform ray that irradiate the flexible beam. The flexible beam is imaged on the photosensitive surface of linear array CCD by objective lens, and then it changes into the electrical signal. The analog-to-digital converters translate it into digital signal, acquire the digital image information by image gathering module card, and save on hardware equipment [4].

Supposing the middle point, the $2 / 3$ point and the terminal point is the marked measuring points A, B, C. the image gathering module card collects the image when the flexible beam is still firstly, then continually collects the vibration images and stores at the computer. Finally, the images are preprocessed in MATLAB, getting the domain response of the flexible beam, the waterfall diagram, the frequency, and the damping ratio. 


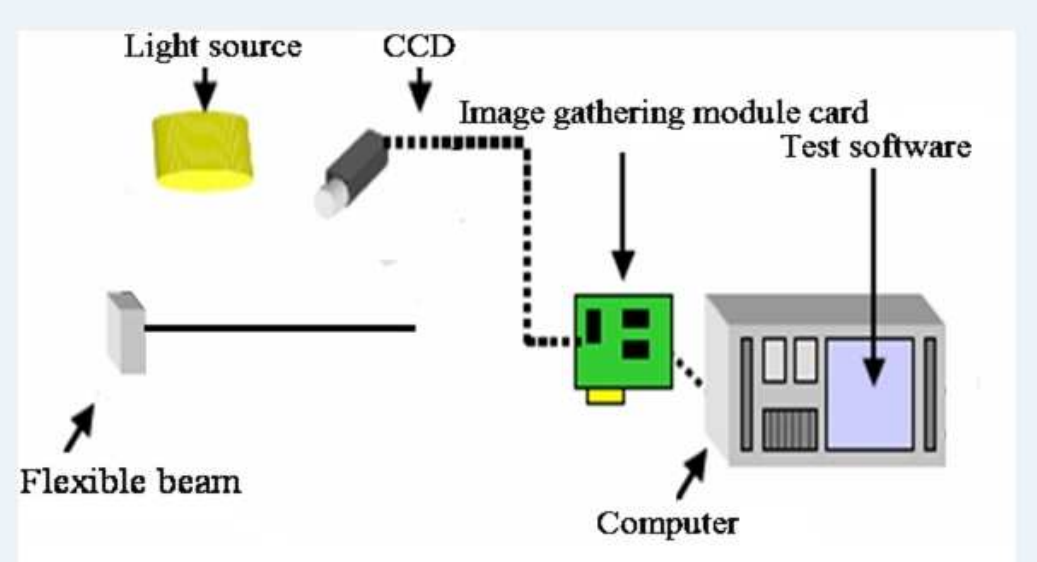

Fig. 1 The principle diagram of flexible beam natural properties test based on CCD

\section{Extraction of marked point coordinates}

The experiment trace, test and record the domain information of displacement by the marked point on the flexible beam. The material of beam is steel 210, and its color tends to black, while the marked point is white, as shown in Fig. 2. The conditions of the marked point is uniform color, clear edge, and the small area, however, it can not be too small so that it is difficult to identify [5].

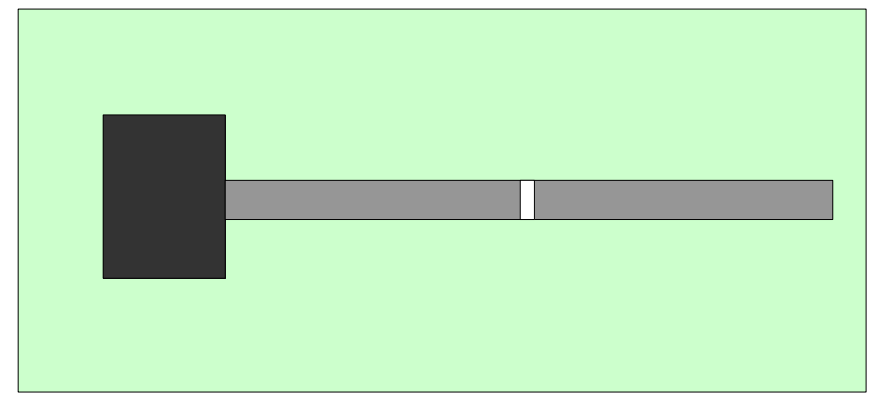

Fig. 2 The marked point of flexible beam

After binary processing of the collected images [6], the marked point becomes clear. Using centroid method extracts the position of marked point. Supposing the gray value is expressed as $f(x, y), 0<x<j, 0<y<j$ the coordinate of centre-of-mass is given as follows:

$$
x_{m}=\frac{\sum_{i j} x f(x, y)}{\sum_{i j} f(x, y)}, y_{m}=\frac{\sum_{i j} y f(x, y)}{\sum_{i j} f(x, y)}
$$

Supposing the coordinate of static marked point $x_{0}$ is $(x, y)$, and the coordinate of exercise marked point is $\left(x^{\prime}, y^{\prime}\right)$ at a certain time. Then the displacement of image $S$ is expressed as follows:

$$
S(x, y)=\left(x-x^{\prime}, y-y^{\prime}\right)
$$

Supposing $a$ the distance between the analyte and the optical center; $R$ is the real displacement of the measured point, and $S$ is the image displacement of linear array CCD. The ratio relation between the measured objects and image can be written as:

$$
R=\frac{S * a}{b}
$$

Inserting $S$ in formula can obtain the vibration displacement $R$. 


\section{Calculations of frequency and damping ratio}

The flexible beam is a system with damping, energy consumption resulting in amplitude decreasing gradually in the process of free vibration. The solution of system with damping is expressed as follows:

$$
x=A \mathrm{e}^{-\zeta \omega_{\mathrm{n}} t} \sin \left(\omega_{\mathrm{d}} t+\varphi\right)
$$

$A$ is the vibration amplitude, $\zeta$ is the damping ratio, $\omega_{\mathrm{n}}$ is the natural frequency of undamped system, and $\omega_{\mathrm{d}}$ is the circular frequency of vibration with damping.

As is shown in formula (3), the vibration of system is not constant amplitude harmonic vibration, however, the amplitude is limited at the range of $\pm A \mathrm{e}^{-\zeta \omega_{\mathrm{n}} t}$, and it decays constantly with time.

$$
T_{\mathrm{d}}=\frac{2 \pi}{\sqrt{1-\zeta^{2}} \omega_{\mathrm{n}}}
$$

Supposing $\zeta=0, \omega_{\mathrm{n}}$ is given as

$$
\omega_{\mathrm{n}}=\frac{2 \pi}{T_{\mathrm{d}}}
$$

Fitting time domain signal attenuation curve with $A \mathrm{e}^{c t}$, the result is found to be

$$
c=-\zeta \omega_{\mathrm{n}}
$$

The frequency and damping ratio can be obtained according to Eqs. (5) and (6).

\section{Test results}

The maximum image resolution is $1292 \times 964$, and the maximum frame rate of camera is $30 \mathrm{fps}$. The type of image acquisition card is DH-VT121 [7]; it has high image quality and stability.

Fig. 3 shows the marked point $\mathrm{C}$ at the end of the flexible beam. The first two collected images of flexible beam are shown in Fig. 4; the first frame is the image at the time $t=0$, the second frame is the image at the time $t=0.03$.

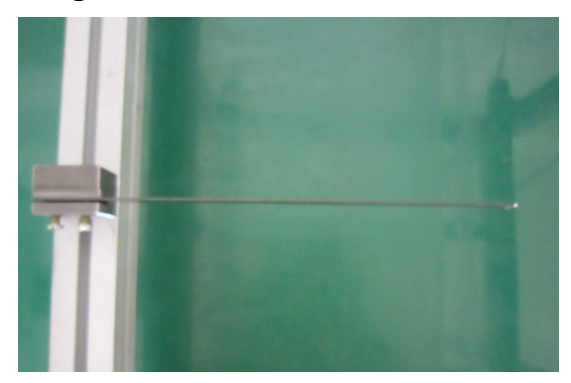

Fig. 3 Flexible beam

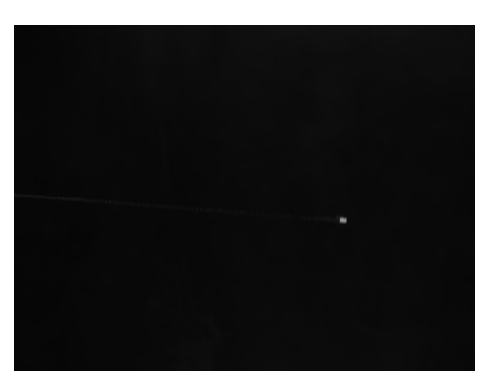

(a) The first frame

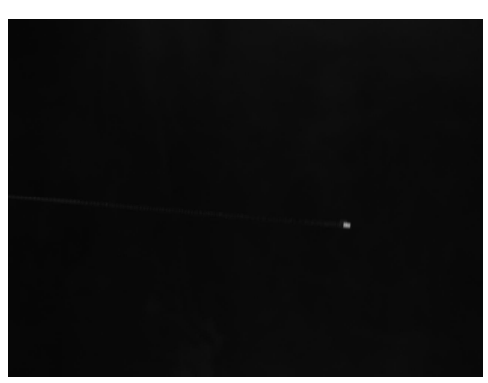

(b) The second frame

Fig. 4 The first two collected images of flexible beam

The collected images shown in Fig. 4 are preprocessed in MATLAB. First, it reads images when the flexible beam is in stationary and dynamic respectively, and makes binary processing of the collected image, then, using centroid method extracts the position of marked point, the image displacement can be obtained by subtracting the image coordinate between the stationary and dynamic images of flexible beam; at last, the domain response of the flexible beam, the waterfall diagram, the frequency, and the damping ratio can be obtained by the converting between image displacement and actual displacement.

The time domain chart and time frequency map can be obtained by the processing above; the results are shown in Fig. 5-Fig. 7, they are expressing the domain and the frequency dynamic responses of the flexible beam marked at point A, point B and point C. From the graph, the amplitude of different marked points is different, and the amplitude of $\mathrm{C}$ point domain response is maximal, then general trend is identical at the point $\mathrm{B}$ and the point $\mathrm{C}$ of the flexible beam. 


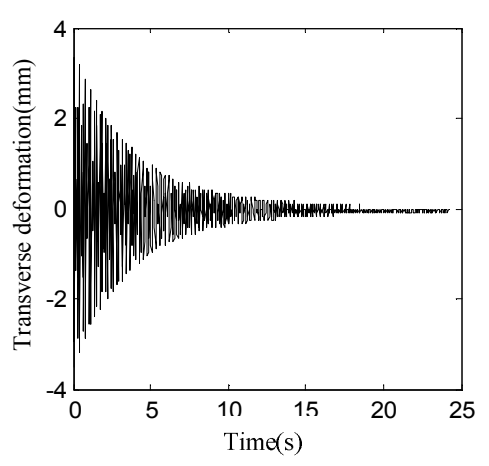

(a) Time domain chart

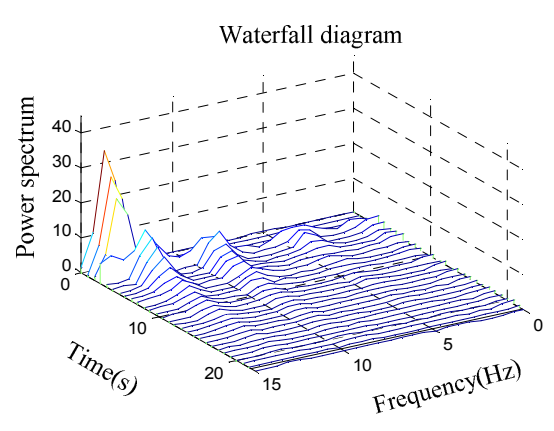

(b) Time frequency map

Fig. 5 Test results of flexible beam at point A

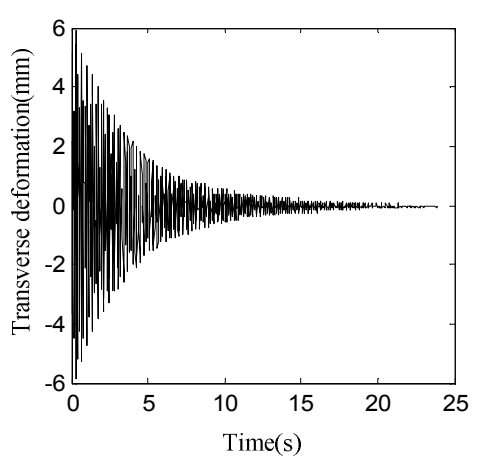

(a) Time domain chart

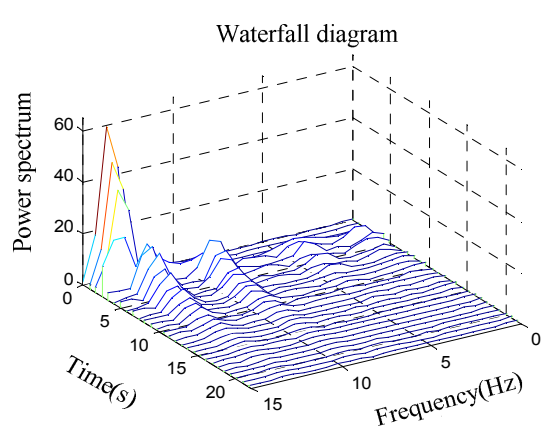

(b) Time frequency map

Fig. 6 Test results of flexible beam at point B

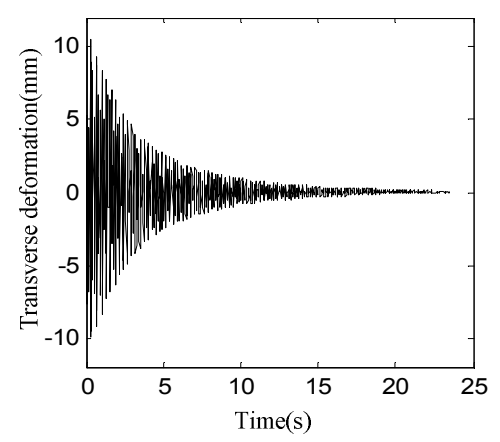

(a) Time domain chart

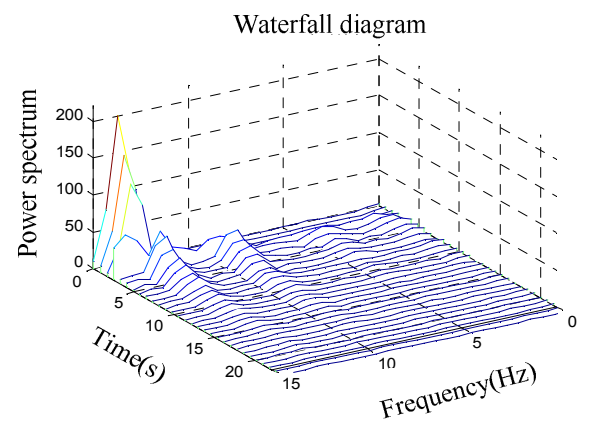

(b) Time frequency map

Fig. 7 Test results of flexible beam at point $\mathrm{C}$

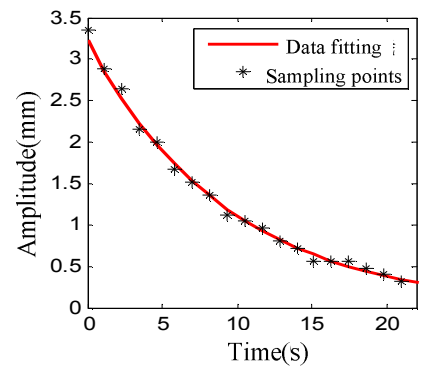

(a) The point $\mathrm{A}$

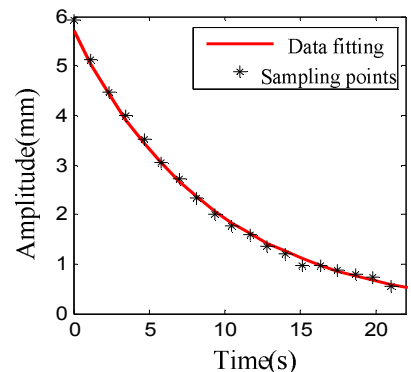

(b) The point B Fig. 8 Data fitting

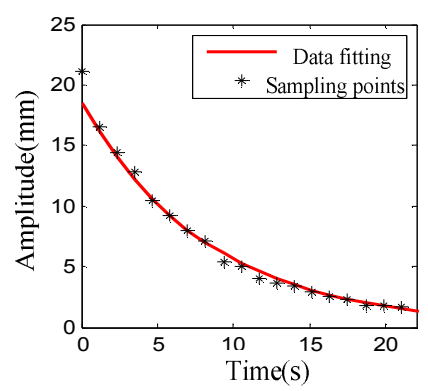

(c) The point $\mathrm{C}$ 
The data fitting between the time domain signal attenuation curve of three marked points and $A \mathrm{e}^{c t}$ is shown in Fig. 8, calculating the frequency and the damping ratio by MATLAB is expressed in Table 1. The numerical results of frequency is 14.21 by theoretical calculation, it is shown that error is very small between image processing technology and theoretical analysis. Moreover, the damping ratio is consistent at the three points, and it meets the experimental requirement.

Table 1 Test results

\begin{tabular}{lllll}
\hline & Point A & Point B & Point C & Average \\
\hline Frequency /HZ & 13.75 & 13.75 & 13.75 & 13.75 \\
Damping ratio & 0.0012 & 0.0013 & 0.0014 & 0.0013
\end{tabular}

\section{Conclusions}

The domain and frequency natural properties of the flexible beam are obtained simulated by force-hammer based on CCD and image processing technology. Binary processing of the collected signal can decrease the environment interference. Centroid method is used to extract the position of marked point. The domain response of the flexible beam, the waterfall diagram, the frequency, and the damping ratio can be got by the converting between image displacement and actual displacement. Though comparing, it is found that the error of frequency is very small, and the damping ratio is consistent at the three points. Thereby test result proves that the testing method has the characteristics of simple operation, high efficiency and high accuracy.

\section{Acknowledgements}

This work was financially supported by the Fundamental Research Funds for the Central Universities (N090603007, N100503001).

\section{References}

[1] J Lin Burn, Vibration Tolerance in Optical Imaging [J]. Optical Engineering, March, 1993, $32(3)$

[2] $\mathrm{T} \mathrm{C} \mathrm{Chu,} \mathrm{et} \mathrm{al.} \mathrm{Applications} \mathrm{of} \mathrm{Digital} \mathrm{Image} \mathrm{Correlation} \mathrm{Techniques} \mathrm{to} \mathrm{Experimental}$ Mechanics [J]. Experimental Mechanics, September, 1985, 25(3).

[3] Kaoli Huang, Changqing Zhu, Application of Liner CCD Sensor. Proceedings of the International Symposium on Test and Measurement, 1999:951-953

[4] Long Qi. The Experiment of Testing Object Vibration by the Linear Array CCD, Laboratory Science, 2006(in Chinese)

[5] Bo Jin. The Research of Bridge Detection and Optic Testing Methods [D], Tianjin: Tianjin university department of solid force, 20049(in Chinese)

[6] Tuoqun Liu. A Non-contact Vibration Measurement Method Based on CCD. Machinery \& Electronics, 2002,(4):76 77(in Chinese)

[7] The Specification of Daheng Image Vision Technology, China Daheng Group Inc. 